\title{
Eisenmangel frühzeitig erkennen und rasch korrigieren
}

Die Prävalenz des Eisenmangels ist bei Patienten mit chronisch-entzündlichen Darmerkrankungen (CED) besonders hoch. Bereits vor einer Anämie bringt der Eisenmangel körperliche Probleme mit sich. Ein regelmäßiges Screening ermöglicht das frühe Einschreiten.

Quelle: Web-Pressekonferenz: „Inside Medicine - Eisenmangel bei CEDPatienten: Einblicke und persönliche Erfahrungen eines Gastroenterologen", 09.06.2020; Veran-

stalter: Vifor Pharma Deutschland $\mathrm{GmbH}$ Manuskript: Ingo Schroeder (ins), Köln gelte es, früh einen Eisenmangel zu erkennen, um rechtzeitig einschreiten zu können, erläuterte Klag das diagnostische Vorgehen. „Liegt ein Eisenmangel vor, sollte auf Grundlage der klinischen Situation eine Supplementation in Betracht gezogen werden", so Klag. Mit der intravenösen Eisengabe können die Eisenspeicher rascher als mit oraler Gabe aufgefüllt werden. Bei neueren Präparaten wie der Eisencarboxymaltose (Ferinject ${ }^{\circledR}$ ) sind zwei Gaben ausreichend, um bei einem manifesten Eisenmangel eine Eisengabe von $1500 \mathrm{mg}$ zu erreichen. Ein weiterer Vorteil der intravenösen Gabe: Sie ist vom Hepcidin-Block und somit von der entzündlichen Aktivität der CED unabhängig. entzündlichen Erkrankungen wie der CED produziert die Leber vermehrt Hepcidin, welches das Ausschleusen von Eisen über das MembrantransportProtein Ferroportin aus der Darmzelle in die Blutbahn blockiert, der sogenannte Hepcidin-Block.

\section{Nicht die Anämie abwarten}

Mithilfe entsprechender Marker wie Ferritin und der Transferrin-Sättigung

\section{Intravenöse Behandlung bei Eisenmangelanämie}

Die Konsensus-Guideline der European Crohn's and Colitis Organisation (ECCO) empfiehlt intravenöses Eisen als Erstbehandlung bei CED-Patienten mit aktiver Erkrankung, bei schwerer Anämie $(\mathrm{Hb}<10 \mathrm{~g} / \mathrm{dl})$, bei vorangegangener Unverträglichkeit gegen-

über oralem Eisen sowie bei Patienten die Erythropoetin erhalten [4].

\section{Literatur \\ 1. Sonnenberg E, Biedermann L (2019) Chronische Schmerzen und Fatigue bei chronisch-entzündlichen Darmerkrankun- gen. Gastroenterologe 14:441-450 \\ 2. Kulnigg S, Gasche C (2006) Systematic Re- view: Managing Anaemia in Crohn's Disease Aliment Pharmacol Ther 24:1507-1523 \\ 3. Gasche $C$ et al (2004) Iron, Anaemia, and Inflammatory Bowel Diseases. Gut 53:1190-1197 \\ 4. Dignass AU et al (2015) European Consensus on the Diagnosis and Management of Iron Deficiency and Anaemia in Inflammatory Bowel Diseases. J Crohns Colitis 9:211-222}

Hinweis des Verlags. Der Verlag bleibt in Hinblick auf geografische Zuordnungen und Gebietsbezeichnungen in veröffentlichten Karten und Institutsadressen neutral.

Schweiz. Gastroenterol. 2020 · 1:108 https://doi.org/10.1007/s43472020-00015-X

C Springer-Verlag GmbH Austria, ein Teil von Springer Nature 2020 